\title{
BMJ Open Comparison of the therapeutic dose of warfarin in HIV-infected and HIV-uninfected patients: a study of clinical practice
}

\author{
B S Jackson, T Mokoena
}

To cite: Jackson BS, Mokoena T. Comparison of the therapeutic dose of warfarin in HIVinfected and HIV-uninfected patients: a study of clinical practice. BMJ Open 2017;7:e013709. doi:10.1136/bmjopen-2016013709

- Prepublication history for this paper is available online. To view these files please visit the journal online (http://dx.doi.org/10.1136/ bmjopen-2016-013709).

Received 2 August 2016 Revised 5 January 2017 Accepted 13 January 2017

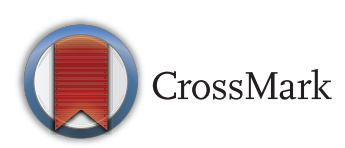

Department of Surgery, University of Pretoria and Steve Biko Academic Hospital, Pretoria, South Africa

Correspondence to T Mokoena;

Taole.Mokoena@up.ac.za

\section{ABSTRACT}

Background: People infected with HIV are prone to venous thrombosis. Treatment of thrombosis is primarily with warfarin. No studies have addressed the effects of HIV infection on warfarin dose. The aims of this study were to determine whether the therapeutic dose of warfarin and induction time to therapeutic dose in HIV-infected patients differ from that in HIVuninfected patients.

Methods: A prospective and retrospective descriptive study of induction time to therapeutic warfarin dose, as well as of ambulant therapeutic warfarin dose, was performed. HIV-infected and HIV-uninfected patients being treated after deep venous thrombosis with or without pulmonary embolism were compared. Sex and use of antiretroviral drugs (ARVs) were also compared in the groups.

Results: 234 patients were entered into the study. Induction time to therapeutic warfarin dose did not differ between the 2 groups. The mean therapeutic dose of warfarin was higher in the HIV-infected than the HIV-uninfected group: 6.06 vs $5.72 \mathrm{mg} /$ day, but this was not statistically significant $(p=0.29)$. There was no difference in therapeutic warfarin dose between ARV-naïve groups-HIV-uninfected and HIV-infected patients not on ARVs.

Conclusions: There appears to be little effect of HIV infection on warfarin dosing. Warfarin therapy should be administered conventionally in HIV-infected patients.

\section{INTRODUCTION}

There is a high incidence of venous thrombosis in patients infected with HIV. The risk is up to 10 times that in uninfected people. $^{1}{ }^{2}$ The aetiology of thrombosis is multifactorial and includes HIV-mediated endothelium activation, malignancies and infections. ${ }^{3}$ The prothrombotic state of HIV infection is also well recognised and several laboratory coagulation parameters have been shown to be abnormal in these patients. ${ }^{4} 5$ While the exact mechanism of this state remains to be fully elucidated, many

\section{Strengths and limitations of this study}

- This is a unique study which investigates the effect of HIV infection on warfarin treatment.

- It is a clinical study of actual management of HIV-infected patients with venous thrombosis.

- For this reason, the study deliberately does not investigate the effect of confounding factors affecting warfarin dosage.

- The authors did not have control over the management of the patients in the retrospective part of the study.

- While the statistical analysis is cogent, a sample size was not predetermined.

coagulation factor abnormalities have been reported, notably protein $\mathrm{C}$, protein $\mathrm{S}$ and antithrombin deficiency. ${ }^{4}$ Patients who have been treated for thrombosis may require long-term anticoagulation primarily with warfarin because the risk of thrombosis in HIV-infected thrombotic patients is not necessarily abolished by antiretroviral treatment and improvement of the CD4 count. ${ }^{4} 6$ Antiretroviral (ARV) drugs per se may also contribute to the hypercoagulable state. ${ }^{7}$ Additionally, these drugs have variable effects on CYP450 liver enzymes that metabolise warfarin and therefore may affect management of thrombosis in HIV-infected patients. ${ }^{8}$ Warfarin has a critical therapeutic window making correct dosing important.

The effect of the HIV status of patients on warfarin dosage has not been investigated. It has been our impression that HIV-infected patients require higher warfarin doses than non-infected patients to achieve therapeutic anticoagulation. Anecdotally longer therapeutic induction times have been observed and this could be due to higher warfarin requirements. Given the possible multiple factors causing hypercoagulability in the HIV-infected patient, we hypothesised that 
these patients with HIV infection may require higher doses of warfarin for induction and maintenance of anticoagulation.

\section{Aim}

The aim of the study was to determine if there is an effect on therapeutic warfarin dose in patients infected with HIV.

The objectives were:

1. To determine whether the therapeutic dose of warfarin differed between groups of HIV-infected and HIV-uninfected patients hospitalised for a thromboembolic incident and followed up at a clinic for international normalised ratio (INR) control.

2. To determine whether the induction time to therapeutic warfarin dose differed between groups of HIV-infected and HIV-uninfected patients.

\section{Motivation}

An observation of apparently increased warfarin requirements by HIV-infected patients hospitalised for venous thrombosis led the authors to investigate the induction time to therapeutic INR in these patients, and to compare this with HIV-uninfected patients. There being no anticoagulation protocol specifically for HIV-infected patients, the comparison would be done using the standard protocol in our department. The induction times to therapeutic INR were to be recorded prospectively and patients followed up at coagulation clinics to determine their ambulant therapeutic warfarin dosage. In order to augment the data, files of additional patients were studied retrospectively at two clinics.

\section{METHODS}

A descriptive study of patients with venous thromboembolism was undertaken. Data were collected both prospectively and retrospectively. Patients aged 18 years and older who had presented with apparently spontaneous lower limb deep venous thrombosis, with or without pulmonary embolism, were included in the study. Patients with attributable causes of thrombosis such as recent surgery, cardiac failure and thrombophilias were excluded. Patients who refused HIV testing were not entered in the study. Confirmation of deep venous thrombosis was by Doppler ultrasound and of pulmonary embolism by CT angiography as indicated. ARV drug treatment as well as the specific drugs was also recorded. Comparisons of the mean daily warfarin dose and induction time to therapeutic warfarin dose were performed in HIV-infected and HIV-uninfected patients, in males and females, and in HIV-infected patients on and not on ARV drug therapy. The use of ARV drugs was recorded and analysed as a group of possible confounders but not all individual drugs were analysed.

- Prospective data collection

All patients admitted to hospital with acute deep venous thrombosis of the lower limb with or without pulmonary embolism were subjected to a consented HIV test and entered in the study. Warfarin dose data were collected during hospitalisation and at follow-up. Clinic visits were scheduled every 2-4 weeks after discharge from hospital. The patients were observed until the attainment of therapeutic warfarin dose, and the number of days recorded. Once induction time had passed, the patients were followed until the therapeutic dose of each patient was attained.

- Retrospective data collection

Warfarin dose data were gathered retrospectively from two follow-up clinics for ambulatory patients after a thromboembolic episode. One clinic was for general anticoagulation control (HIV-uninfected patients), while the other was for HIV-infected patients for AIDS follow-up (HIV-infected patients). The latter patients were included irrespective of the duration of HIV infection. Only patients who attended clinics regularly until the therapeutic dose was achieved were included in this study. Induction time was computed and therapeutic warfarin dose recorded.

\section{Definitions}

\section{Warfarin regimen}

The same regimen was used for all patients in the study. Sodium-warfarin (Cipla-Warfarin, Cipla-Medpro) was started at $5 \mathrm{mg}$ per day and followed by a modification of the slow Fennerty regimen, using dose adjustments of $2.5 \mathrm{mg} .{ }^{9}$ The INR was determined every $2-3$ days after initiation or change of treatment dose, while maintaining anticoagulation with a low molecular weight heparin until an INR level of between 2 and 3 was obtained.

\section{Warfarin induction time}

The induction time to therapeutic warfarin dose was defined as the time in days to achieve an INR measurement of between 2 and 3 . The number of days was calculated as the number of days from the start of warfarin therapy until the day of stable therapeutic dose (the first day of consecutive identical doses). Arithmetic means of the number of days were calculated for groups of patients.

\section{Therapeutic warfarin dose}

The target INR for ambulant warfarin therapy was 2.5, with a range of 2-3. The last warfarin dose recorded after dosage stabilisation at the INR target range of 2-3 had been achieved was recorded as the therapeutic daily dose for the prospectively and retrospectively studied patients. If the dose differed on alternate days, the average of 2 days' dosages was recorded. Means of ambulant warfarin dose of groups of patients were calculated for comparison. Owing to the short-term goals of this study of recording only induction time and the most recent therapeutic warfarin dose, time in therapeutic range was not determined.

The study was conducted at the Pretoria Academic Health Complex consisting of the Steve Biko Academic Hospital and Kalafong Regional Hospital, as well as the 
Table 1 Demographics of study patients with or without HIV infection on warfarin therapy, $n=234$

\begin{tabular}{llllll}
\hline & Males & Females & CD4 cell count (mean) ${ }^{\star}<\mathbf{2 0 0 ,}>\mathbf{2 0 0}$ & M/Ft & Mean age years (range) \\
\hline HIV $+n=112$ & 44 & 68 & 35,48 & $43 / 41$ & $41.9(20-71)$ \\
HIV $-n=122$ & 42 & 80 &,-- & $49 / 47$ & $47.8(18-83)$ \\
Total $n=234$ & 86 & 148 & & &
\end{tabular}

${ }^{*} \mathrm{CD} 4$ count unknown $=29$ patients.

$+\mathrm{M} / \mathrm{F}=$ ratio of males to females.

HIV+, HIV infected; HIV-, HIV uninfected; F, females; M, males.

Tshwane District Hospital, during the period 2013-2015. Individual consent was obtained from the prospectively studied patients.

The study is reported in accordance with the STROBE criteria. $^{10}$

\section{Statistical analysis}

The two-sample t-test of the comparison of means of warfarin dose was performed in the following groups: HIV-infected and HIV-uninfected patients, HIV-infected patients on and not on ARV drugs, and males and females in these groups. Sex and the use of ARV drug use were analysed as possible confounders. Induction times for the groups to attain therapeutic warfarin doses were analysed by the Kruskal-Wallis test. The study was considered to be exploratory in nature. Since the difference in warfarin dose outcomes of the study groups was expected to be small, sample size and size effect were not predetermined as a large number of patients would be required. A p value of 0.05 was taken to be significant.

\section{RESULTS}

Patient demographics and the number of patients in each group are depicted in table 1 . The age of the patients was somewhat lower in the HIV-infected group.

\section{Induction time to therapeutic warfarin dose}

Induction times were analysed in 170 patients. The data were collected prospectively for 93 patients and retrospectively for 77 patients. Seventy-four of the patients were HIV-infected and 96 HIV-uninfected. The mean times were 12.87 and 11.19 days, respectively $(p=0.28)$. Thirty-six of the HIV-infected patients were on ARV drugs. Their induction times did not differ significantly from those of the HIV-infected patients not on ARV drugs or the HIV-uninfected patients (figure 1). It must be noted that therapeutic induction times exhibited a wide range of SD.

\section{Stable ambulant warfarin dose}

The prospectively studied patients in the warfarin induction study were followed up for determination of their ambulant therapeutic warfarin dose. These 93 patients were augmented by data collected retrospectively from 141 patients. This gave a total of 234 patients, 122 HIV-uninfected (42 males and 80 females) and 112 HIV-infected (44 males and 68 females; table 2). In general, males required more warfarin than females. This applied to all groups of patients but only the comparison for HIV-uninfected patients was significant: males $(6.49 \mathrm{mg})$ and females $(5.31 \mathrm{mg} ; \mathrm{p}=0.01)$.

The mean therapeutic dose of warfarin in the whole set of patients was higher in the HIV-infected group
Figure 1 Induction time to therapeutic warfarin dose in days $\pm 1 \mathrm{SD}$ in patients with (HIV+) or without HIV (HIV-) infection and on antiretroviral (ARV) therapy $(A R V+)$ or $A R V$ therapy naïve (ARV-).

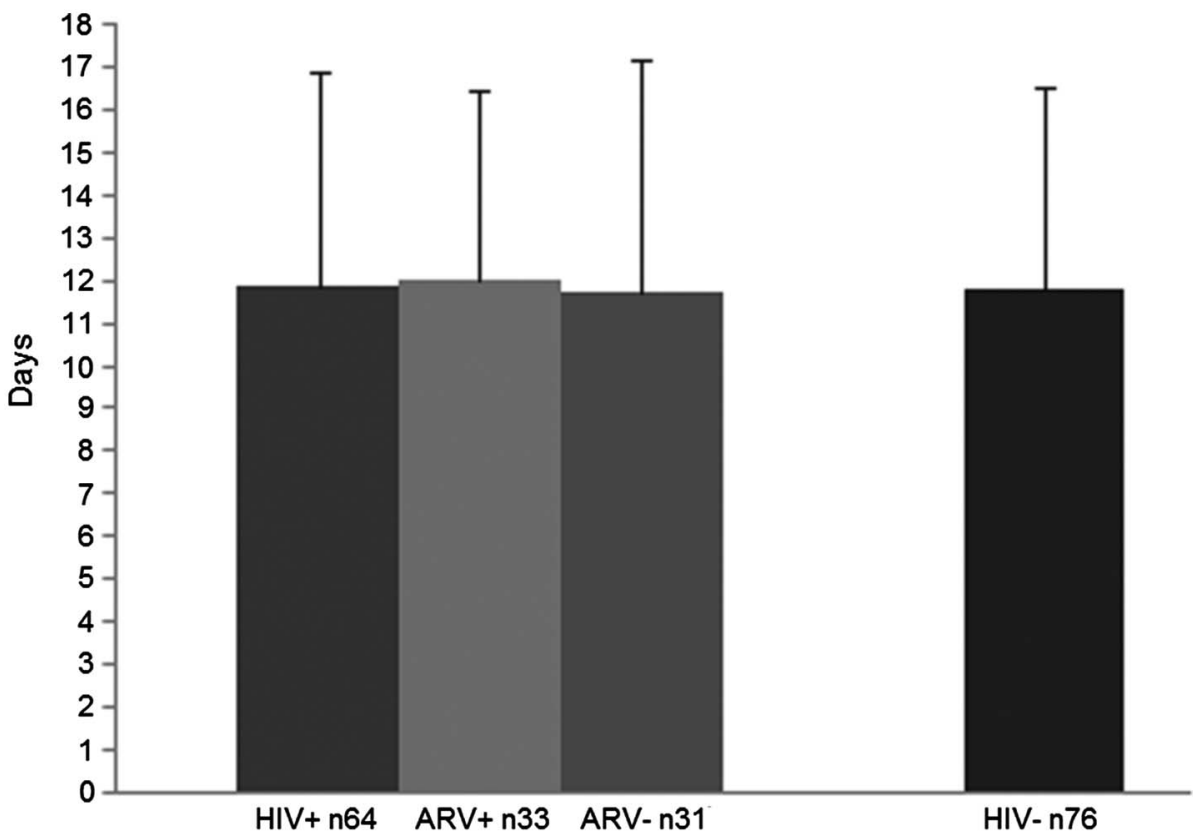


Table 2 Mean warfarin doses (mg/day) according to patients' HIV infection and ARVT status

\begin{tabular}{|c|c|c|}
\hline Patient groups (n, \%) & $\begin{array}{l}\text { Warfarin } \\
\text { dose } \\
\text { (mg/day) }\end{array}$ & p Value \\
\hline \multicolumn{3}{|l|}{ All $(234,100)$} \\
\hline HIV+ $(112,48)$ & 6.06 & $p=0.29$ \\
\hline HIV- $(122,52)$ & $5.72^{*}$ & 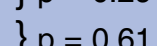 \\
\hline $\mathrm{HIV}+$ and ARVT- $(62,55)$ & $5.94^{*}$ & \\
\hline HIV+ and ARVT+ $(50,45)$ & 6.20 & $p=0.59$ \\
\hline \multicolumn{3}{|l|}{ Male $(86,100)$} \\
\hline HIV+ $(44,51)$ & $6.39 \dagger$ & $p=0.87$ \\
\hline HIV- $(42,49)$ & $6.49 \ddagger$ & $n=0.63$ \\
\hline HIV+ and ARVT- $(22,50)$ & $6.92 \S$ & 0.03 \\
\hline HIV+ and ARVT+ $(22,50)$ & 5.879 & $p=0.23$ \\
\hline \multicolumn{3}{|l|}{ Female (148) } \\
\hline HIV+ $(68,46)$ & $5.84 \dagger$ & $p=0.14$ \\
\hline HIV- $(80,54)$ & $5.31 \ddagger$ & $\mathrm{p}=0.84$ \\
\hline HIV+ and ARVT- $(40,58)$ & $5.40 \S$ & $p=0.84$ \\
\hline $\mathrm{HIV}+$ and $\mathrm{ARVT}+(28,42)$ & 6.479 & $p=0.06$ \\
\hline
\end{tabular}

${ }^{*}$ Comparison of ARVT-naïve groups, $p=0.22$.

†Male versus female, $p=0.29$.

$\ddagger$ Male versus female, $p=0.01$.

§Male versus female, $\mathrm{p}=0.09$.

qMale versus female, $p=0.3$.

ARVT, antiretroviral drug therapy. warfarin than those not on ARV drugs, 6.47 vs $5.40 \mathrm{mg} /$ day $(p=0.06)$. However, HIV-infected females on ARV drugs required significantly more warfarin, $6.47 \mathrm{mg} /$ day, than HIV-uninfected females, $5.31 \mathrm{mg} /$ day $(p=0.01)$. The opposite was true for males, but the difference was not statistically significant, 5.87 vs $6.49 \mathrm{mg} /$ day $(\mathrm{p}=0.29)$.

ARV-naïve patient groups were compared. There was no significant difference in warfarin dose between HIV-infected patients not taking ARV drugs and HIV-uninfected patients, 5.94 vs $5.72 \mathrm{mg} /$ day $(\mathrm{p}=0.22$; table 2).

Most of the individual ARV drugs were taken by few patients. Eleven patients were taking stavidine and fewer were on several other drugs (table 3 ). In addition, drugs were taken in various different combinations, making analysis of individual drug effects on warfarin dose unrealistic. However, three drugs were taken by substantially more patients, efavirenz, lamivudine and zidovudine. The mean warfarin dose of the 22 patients taking efavirenz (a CYP450 inhibitor) was virtually the same as that of the HIV-uninfected patients (5.73 and 5.72, respectively). Twenty-five and 24 patients, respectively, were taking zidovudine and lamivudine, which are not metabolised by the CYP450 pathway and apparently do not affect warfarin blood levels.

\section{DISCUSSION}

We report on a comparative study of warfarin dosage in HIV-infected and HIV-uninfected patients. This comparison, using a standard warfarin treatment protocol, has not been published previously. The assumption that HIV-infected patients require higher doses of warfarin for therapeutic effect seems to be reasonable given the acknowledged hypercoagulable state of the HIV-infected patient. Anecdotal observation in our practice suggested that this is indeed the case. Many of these patients had not yet started ARV therapy. Previous warfarin dosage studies in HIV-infected patients have largely addressed the effect of ARV drugs on warfarin dose. ${ }^{4} 6711$ This study attempted to determine the possible role of HIV infection. However, no apparent increase in warfarin requirements for anticoagulation was demonstrated in HIV-infected patients compared with uninfected patients in general, except in females on ARV therapy.

There is a paucity of clinical studies on the effect of infection in general on warfarin dosage. This has been addressed in a few studies on patients with acute infection. ${ }^{12} 13$ The effect of HIV infection on warfarin dosage has not been similarly studied. Infection with HIV, quite apart from the effect of concomitant infections and malignancies and various possible drug interactions, apparently causes a propensity for thrombosis, as recovery of the CD4 count does not abolish the increased risk of thrombosis. ${ }^{246}$ The current study attempted to determine indirectly the effect of the HIV infection per se on warfarin requirement by comparison of the dosage with that in HIV-uninfected patients. The mean warfarin 
dosage was found not to differ between these two broad groups.

Clinical management of warfarin therapy is difficult because of interindividual and intraindividual variability of dosage requirements. ${ }^{14}{ }^{15}$ The narrow therapeutic window for warfarin dosage makes accurate dosing important. ${ }^{16}$ Many patient and environmental factors influence warfarin dosing. These include sex, race, height, weight and age. ${ }^{14}{ }^{15}$ Only the known increased warfarin requirements for men were documented and are apparent in this study, in the comparison of ARV-uninfected men and women.

Warfarin dose effects of ARV drugs in HIV-infected patients have been investigated by several authors. ARV drugs have variable effects on warfarin blood levels, some causing an increase in levels and some a decrease. Sekaggya et $a l^{17}$ demonstrated this in a case series of HIV-infected patients being treated for tuberculosis. Jong et $a l^{4}$ demonstrated lower levels of the von Willebrand factor, factor VIII, D-dimer and endogenous thrombin potential in HIV-infected patients on ARV drugs. Anderson $e t a l^{18}$ found that patients on efavirenzbased regimens required lower weekly warfarin doses than patients on lopinavir/ritonavir regimens. This was not the case in this study in the patients taking efavirenz. The warfarin dose in the 22 HIV-infected patients taking efavirenz did not differ from that in the patients not taking the drug. However, this could have been due to other influences not studied here, such as the effects of other drugs. Stolbach et al caution that efavirenz and nevirapine may affect therapeutic INR levels, and that warfarin dose adjustments may be required. Little can be deduced from the ARV drug effect found in this study as the effects of most of the individual ARV drugs on warfarin requirements were not analysed statistically. Patients were taking several ARV drugs singly or in various combinations. The sample size of patients on each drug was too small to meaningfully perform separate analyses. An important comparison in this study, however, is that between the 62 HIV-infected patients not taking ARV drugs and the 122 HIV-uninfected patients. This comparison would discount the effects of the ARV drugs. While there was a modest difference in warfarin dosage, this did not differ significantly between these two groups $(\mathrm{p}=0.20$, table 2$)$. Once again, this may be due to factors not addressed in this study.

The development of a warfarin dosing algorithm for HIV-infected persons would be difficult and complex because of the multiple influences that may be operating. ${ }^{14} 17$ These include genetic factors, the hypercoagulable state, pertinent infections and malignancies, and the effect of multiple drugs on the cytochrome $\mathrm{P} 450$ 2C9 system. These diverse effects on warfarin dosage are probably reflected in the wide range of SD in this study as shown in figure 1 . However, the study addresses the broad group of HIV-infected patients, and does not take cognisance of the multiple factors that can affect warfarin dosing. We propose that this approach is valid because the study reflects clinical usage in which standard warfarin regimens are used for HIV-infected patients. Current practice in HIV-infected patients is to manage warfarin dosing as in HIV-uninfected patients, and this study would seem to support this custom. ${ }^{6}$ While not statistically significant, cognisance should be taken of the moderately higher doses needed for therapeutic warfarin effect in HIV-infected patients. This difference might prove to be clinically significant in a larger study, and one that includes the study of warfarin toxicity due to inaccurate dosing.

The study has some shortcomings. Retrospective data were collected from several sources over which the investigators did not have control. In addition, rates of satisfactory therapeutic anticoagulation have been reported to be low in HIV-infected patients, partly attributable to poor adherence to warfarin therapy. ${ }^{18}$ HIV-infected patients often take ARV drugs as well as other drugs for AIDS-related illnesses such as azole antifungal agents. The drugs have variable effects on warfarin metabolism and may alter warfarin requirements. These effects were not investigated in this study. The sample size of the study was not predetermined. While the statistical results are cogent, it is possible that a type II error occurred in analysis of the data. The study nevertheless suggests the performance of a larger study.

\section{Summary}

This is the first study investigating HIV infection status on warfarin dosing. This was done by comparison with uninfected patients using a standard warfarin administration protocol. Overall, there was no significant statistical difference between these groups either in induction time to therapeutic warfarin dose or stable doses in ambulant patients. However, a subgroup of females with HIV infection on ARV drugs required significantly more warfarin for therapeutic effect. A larger study may detect a difference in warfarin requirements of HIV-infected patients.

\section{CONCLUSION}

It is recommended that warfarin dosing and coagulation monitoring be the same in the routine management of HIV-infected patients as for HIV-uninfected patients.

Acknowledgements The authors would like to acknowledge the assistance of Professor VOL Karusseit of the Department of Surgery of the University of Pretoria in the preparation of the manuscript.

Contributors TM originated the study concept. BSJ did the research which TM supervised. Both contributed to the interpretation of data and manuscript writing.

Funding This research received no specific grant from any funding agency in the public, commercial or not-for-profit sectors.

\section{Competing interests None declared.}

Ethics approval Research Ethics Committee of the Faculty of Health Sciences of the University of Pretoria.

Provenance and peer review Not commissioned; externally peer reviewed. 
Data sharing statement No additional data are available.

Open Access This is an Open Access article distributed in accordance with the Creative Commons Attribution Non Commercial (CC BY-NC 4.0) license, which permits others to distribute, remix, adapt, build upon this work noncommercially, and license their derivative works on different terms, provided the original work is properly cited and the use is non-commercial. See: http:// creativecommons.org/licenses/by-nc/4.0/

\section{REFERENCES}

1. Rasmussen LD, Dybdal M, Gerstoft J, et al. HIV and risk of venous thromboembolism: a Danish nationwide population-based cohort study. HIV Med 2011;12:202-10.

2. Bibas M, Biava G, Antinori A. HIV-associated venous thromboembolism. Mediterr J Hematol Infect Dis 2011 3:e2011030

3. Kiser KL, Badowski ME. Risk factors for venous thromboembolism in patient with human immunodeficiency virus infection. Pharmacotherapy 2010;30:1292-302.

4. Jong E, Louw S, Meijers JC, et al. The hemostatic balance in HIV-infected patients with and without antiretroviral therapy: partial restoration with antiretroviral therapy. AIDS Patient Care STDS 2009:23:1001-7.

5. Funderburg NT, Lederman MM. Coagulation and morbidity in treated HIV infection. Thromb Res 2014;133(Suppl 1):S21-524.

6. Crum-Cianflone NF, Weekes J, Bavaro M. Thrombosis among HIV-infected patients during the highly active antiretroviral therapy era. AIDS Patient Care STDS 2008;22:771-8.

7. Liedtke MD, Rathbun RC. Drug interactions with antiretrovirals and warfarin. Expert Opin Drug Saf 2010;9:215-23.
8. Stolbach A, Paziana $\mathrm{K}$, Heverling $\mathrm{H}$, et al. A review of the toxicity of HIV medications II: interactions with drugs and complementary and alternative medicine products. J Med Toxicol 2015;11:326-41.

9. Fennerty A, Dolben J, Thomas $\mathrm{P}$, et al. Flexible induction dose regimen for warfarin and prediction of maintenance dose. BMJ 1984;288:1268-70.

10. von Elm E, Altman DG, Egger M, et al. Strengthening the Reporting of Observational Studies in Epidemiology (STROBE) statement: guidelines for reporting observational studies. BMJ 2007;335:806-8.

11. Dionisio D, Mininni S, Bartolozzi D, et al. Need for increased dose of warfarin in HIV patients taking nevirapine. AIDS 2001;15:277-8.

12. Schelleman $\mathrm{H}$, Bilker WB, Brensinger $\mathrm{CM}$, et al. Warfarin with fluoroquinolones, sulfonamides, or azole antifungals: interactions and the risk of hospitalization for gastrointestinal bleeding. Clin Pharmacol Ther 2008;84:581-8.

13. Clark NP, Delate T, Riggs CS, et al. Warfarin interactions with antibiotics in the ambulatory care setting. JAMA Intern Med 2014;174:409-16.

14. Jonas DE, Mcleod HL. Genetic and clinical factors relating to warfarin dosing. Trends Pharmacol Sci 2009;30:375-86.

15. Lee MTM, Klein TE. Pharmacogenetics of warfarin: challenges and opportunities. J Hum Genet 2013;58:334-8.

16. Garcia $D$, Regan $S$, Crowther $M$, et al. Warfarin maintenance dosing patterns in clinical practice. Implications for safer anticoagulation in the elderly population. Chest 2005;127:2049-56.

17. Sekaggya C, Nalwanga D, Von Braun A, et al. Challenges in achieving a target international normalized ratio for deep vein thrombosis among HIV-infected patients with tuberculosis: a case series. BMC Hematol 2016;16:16.

18. Anderson AM, Chane T, Patel M, et al. Warfarin therapy in the HIV medical home model: low rates of therapeutic anticoagulation despite adherence and differences in dosing based on specific antiretrovirals. AIDS Patient Care STDS 2012;26:454-62. 